\title{
ASSESSMENT OF VENTRAL TEGMENTAL AREA-PROJECTING GABAERGIC NEURONS FROM THE BED NUCLEUS OF THE STRIA TERMINALIS IN MODULATING BINGE-LIKE ETHANOL INTAKE
}

\author{
Michel A. Companion
}

\begin{abstract}
A thesis submitted to the faculty at the University of North Carolina at Chapel Hill in partial fulfillment of the requirements for the degree of Master of Psychology and Neuroscience in the Behavioral and Integrative Neuroscience program in the College of Arts and Sciences.
\end{abstract}

Chapel Hill

2018

Approved by:

Todd E. Thiele

Kathyrn Reissner

Donald Lysle 
(C) 2018

Michel A. Companion ALL RIGHTS RESERVED 


\begin{abstract}
Michel A Companion: Assessment of ventral tegmental area-projecting GABAergic neurons from the bed nucleus of the stria terminalis in modulating binge-like ethanol intake (Under the direction of Todd E. Thiele)
\end{abstract}

Corticotropin-releasing factor (CRF) circuitry is a key component in plasticity underlying the transition to ethanol $(\mathrm{EtOH})$ dependence. We have previously shown that chemogenetic silencing of CRF neurons stemming from the dorsolateral bed nucleus of the stria terminalis (dlBNST) and projecting to the ventral tegmental area (VTA) significantly blunts binge-like EtOH consumption. While CRF neurons in the BNST are thought to entail primarily a gamma- Aminobutyric acid (GABA) phenotype, glutamatergic neurons within the BNST also innervate the VTA and influence consummatory behaviors. Here we combined the well- validated Vgat- ires-Cre transgenic mice with chemogenetic tools to extend our previous findings and corroborate the contribution of the VTA-projecting dlBNST GABAergic circuitry in modulating binge-like EtOH consumption using "drinking-in-thedark" (DID) procedures. Mice were given bilateral injection of Gi-coupled chemogenetic viral vector (or control virus) into the dIBNST and bilateral cannulae into the VTA. On test day, clozapine-n-oxide (CNO; or vehicle) were infused directly into the VTA to silence VTAprojecting dlBNST neurons and subsequent binge- like EtOH consumption was assessed. We then used immunohistochemistry (IHC) to determine the co-expression of CRF and viral vector. Our results showed that relative to vehicle treatment or $\mathrm{CNO}$ treatment in mice expressing the control virus, silencing VTA- projecting dlBNST GABAergic neurons by CNO treatment in mice expressing Gi-coupled chemogenetic virus significant reduced binge- 
like EtOH intake. This effect was not seen with sucrose consumption. Our IHC results confirm a population of CRF-expressing GABAergic neurons within the dlBNST. This study directly establishes that VTA-projecting GABAergic neurons of the dIBNST modulate bingelike EtOH consumption. 


\section{ACKNOWLEDGEMENTS}

Countless thanks to the Thiele Laboratory, and to my advisor, Todd E. Thiele, whose direction, support, and editing were instrumental in finalizing these experiments and writing this thesis. 


\section{TABLE OF CONTENTS}

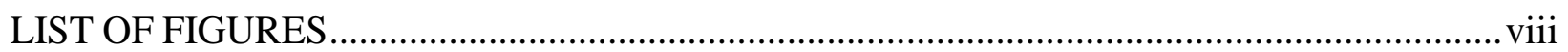

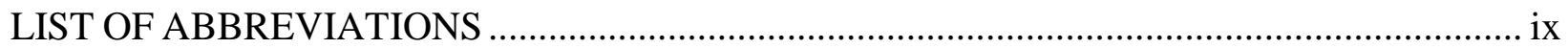

CHAPTER 1: ASSESSMENT OF VENTRAL TEGMENTAL

AREA-PROJECTING GABAERGIC NEURONS FROM THE BED

NUCLEUS OF THE STRIA TERMINALIS IN MODULATING

BINGE-LIKE ETHANOL INTAKE PREFERENCE ..............................................................

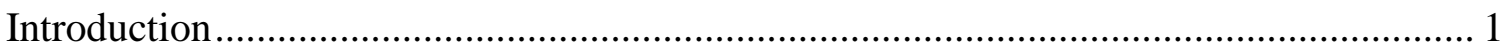

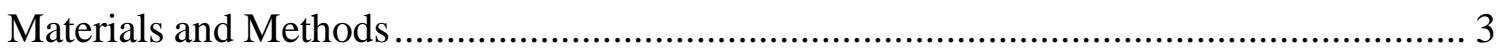

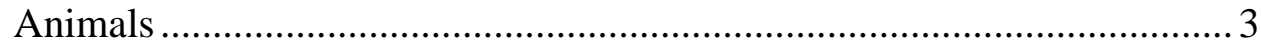

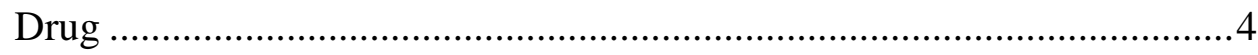

Surgical Procedures................................................................................

Drinking-in-the-dark procedures.............................................................

Tissue Preparation and IHC Procedures.....................................................

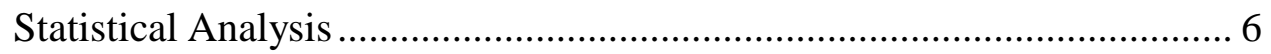

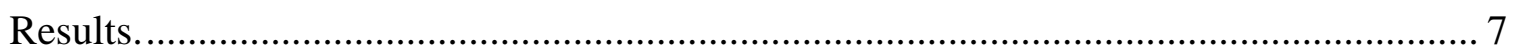

Anterograde Tracing of fibers Reveals VTA-projecting

GABAergic Pathway Stemming from the dlBNST ....................................

Inhibition of GABAergic VTA-projecting dlBNST

Neurons Reduces Binge-like Ethanol Consumption.................................... 8

Inhibition of GABAergic VTA-projecting dlBNST

neurons does not Influence Sucrose Consumption .................................... 10 
Co-localization of CRF immunoreactivity with Cre-dependent DREADD in in the dIBNST of VGat-ires-Cre mice.......................11

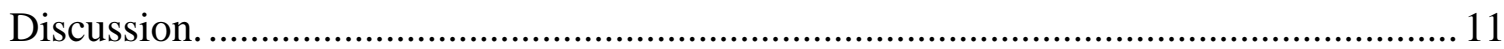

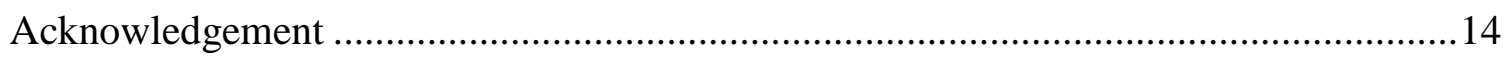

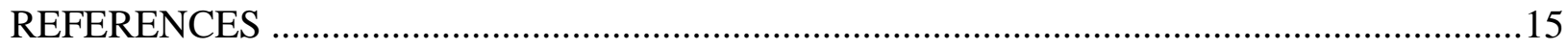




\section{LIST OF FIGURES}

Figure 1 - Tracing of GABAergic neuron terminals from the dIBNST to the VTA within the VGat-ires-Cre mouse line

Figure 2 - Silencing of VTA-projecting dlBNST GABAergic neurons reduces binge drinking

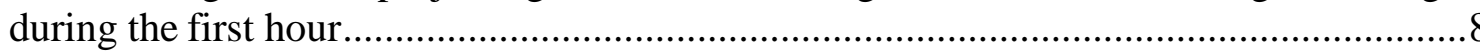

Figure 3 - Silencing of VTA-projecting dlBNST GABAergic neurons had no significant effect on binge drinking of $3 \%$ sucrose ............................................................. 10

Figure 4 - CRF IHC co-localization with Cre-dependent AAV8-hSyn-DIO-hM4d-mCherry in

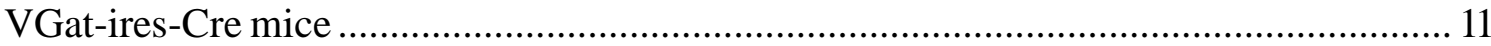




\section{LIST OF ABBREVIATIONS}

$\begin{array}{ll}\text { AAV } & \text { adeno-associated virus } \\ \text { BNST } & \text { bed nucleus of the stria terminalis } \\ \text { CNO } & \text { clozapine- } N \text {-oxide } \\ \text { CRF } & \text { corticotropin-releasing factor } \\ \text { CRF1R } & \text { corticotropin-releasing factor } 1 \text { receptor } \\ \text { CRF2R } & \text { corticotropin-releasing factor } 2 \text { receptor } \\ \text { DID } & \text { Drinking-in-the-dark } \\ \text { dIBNST } & \text { dorsal lateral bed nucleus of the stria terminalis } \\ \text { DMSO } & \text { dimethyl sulfoxide } \\ \text { DREADD } & \text { designer receptors exclusively activated by designerdrugs } \\ \text { eYFP } & \text { enhanced yellow fluorescent protein } \\ \text { IHC } & \text { immunohistochemistry } \\ \text { VGAT } & \text { vesicular } \gamma \text {-Aminobutyric acid transporter } \\ \text { RM } & \text { ventral tegmental area } \\ \text { VANO } & \end{array}$




\section{CHAPTER 1: ASSESSMENT OF VENTRAL TEGMENTAL AREA-PROJECTING GABAERGIC NEURONS FROM THE BED NUCLEUS OF THE STRIATERMINALIS IN MODULATING BINGE-LIKE ETHANOL INTAKE ${ }^{1}$}

\section{Introduction}

It has been established that the method and rate of alcohol consumption contributes to the susceptibility of transitioning from moderate alcohol (ethanol; EtOH) drinking to the development of alcohol dependence (Li et al., 2007). A pattern of short bouts involving excessive alcohol consumption, referred to as binge drinking, is a major proponent of this transition. According to the National Institute of Alcohol Abuse and Alcoholism (NIAAA), binge drinking is consuming enough alcohol to achieve blood concentrations in excess of 80 $\mathrm{mg} / \mathrm{dl}$ in a short period of time (NIAAA, 2004). The "Drinking in the dark" (DID) model has been shown induce binge-like levels of ethanol intake using a restricted access paradigm in C57BL/6J mice (Rhodes et al., 2005; Thiele et al., 2014; Thiele \& Navarro, 2014). Utilizing this approach to study system neurocircuitry has helped provide insight to the underlying mechanisms involved with binge consumption and the transition from moderate alcohol use to dependence.

One neuropeptide system heavily involved in both binge-like EtOH intake and dependence-induced drinking is the corticotropin-releasing factor (CRF) system (Lowery \& Thiele, 2010; Roberto et al., 2010; Lowery-Gionta et al., 2012).

\footnotetext{
${ }^{1}$ This chapter is under review as an article in the journal European Journal of Neuroscience. The original citation is as follows: Companion, M.A. \& Thiele, T.E. under review. Assessment of ventral tegmental area-projecting GABAergic neurons from the bed nucleus of the stria terminalis in modulating binge-like ethanol intake. European Journal of Neuroscience.
} 
Previous work has shown that CRF protein levels within the ventral tegmental area (VTA) become elevated after exposure to binge-like EtOH consumption (Lowery-Gionta et al., 2012). More recently, our laboratory has discovered that the inhibition of CRF axonal projections stemming from the dorsal lateral BNST (dlBNST) and innervating the VTA leads to a decrease in binge-like EtOH consumption in transgenic CRF-ires-Cre mice on a C57BL/6J background (Rinker et al., 2017). These CRF projection neurons have been shown to exert a GABAergic phenotype (Dabrowska et al., 2013). Consistently, GABA-A receptor inhibition in the posterior VTA has been shown to reduce binge-like EtOH consumption in C57BL/6J mice (Melón \& Boehm, 2011). However, there are also other populations of neurons in the BNST that innervate the VTA, such as glutamatergic neurons, that have also been shown to be involved in consummatory behaviors (Kudo et al., 2012; Jennings et al., 2013; Stamatakis et al., 2014). The established co-expression of GABA and CRF in dlBNST neurons suggest that our previous results involved GABAergic neurons, though the direct assessment of VTA-projecting GABAergic neurons of the dlBNST in the modulating of binge-like EtOH has not been directly tested.

The goal of the present study was to confirm that our previous study, using CRF-ires-Cre mice, likely involved a GABAergic neuronal population housing this CRF peptide. To this end we used well-established transgenic mice in which Cre was linked to the vesticular GABA transporter, VGAT (the Vgat-ires-Cre line, (Vong et al., 2011)) in combination with Credependent adeno-associated viral vectors (AAVs) that were infused into the dlBNST of Vgatires-Cre mice. This chemogenetic technology that we employed has been referred to as Designer Receptors Exclusively Activated by Designer Drugs (DREADD) and acts by producing Gcoupled protein receptors, reactive to naturally inert ligands, such as Clozapine-n-oxide (CNO) (Rogan \& Roth, 2011; Roth, 2016). In addition to bilateral infusion of Cre-dependent Gi/o 
DREADD (or control virus lacking the DREADD construct) into the dlBNST, Vgat-ires-cre mice were simultaneously given bilateral cannulae aimed at the VTA. By infusing CNO specifically into the VTA we were able to specifically inhibit VTA-projecting GABAergic neurons arising from the dlBNST area where the virus was injected. We then applied immunohistochemistry (IHC) to assess co-expression of CRF with our DREADD virus in dlBNST neurons.

\section{Materials and Methods}

Experiments were conducted in accordance with the NIH Guide for the Care and Use of Laboratory Animals. All procedures were reviewed and approved by the Institutional Animal Care and Use Committee (IACUC) of the University of North Carolina at Chapel Hill.

Animals. This study used both male and female vGat-ires-Cre mice (determined Cre+ by standard PCR genotyping) backcrossed with the C57BL/6J (Jackson Laboratories, Bar Harbor, MA, USA), which have previously been shown to consume high binge-like levels of EtOH in a restricted consumption model (Crabbe et al., 2011). Mice were at least 60 days of age before the beginning of this study. Animals were individually housed in a ventilated cage system on a 12hour reversed light/dark cycle with ad libitum access to Prolab ${ }^{\circledR}$ RMH 3000 (Purina labDiet ${ }^{\circ}$; St. Louis, MO) and water during the duration of the experiment; except for during EtOH access periods (Marshall et al., 2015). A total of $\mathrm{N}=44$ mice were used in this study with individual group n's listed in figures. All mice within the sucrose portion of the experiment overlap with a portion from the EtOH trials. A separate group of $n=6$ animals, half male and half female, were used in order to quantify overlap of CRF+ and DREADD-expressing neurons within the dlBNST region of viral injection.

Drug. Ethanol (20\% v/v) solutions were prepared from 95\% ethyl alcohol stock (Deacon Laboratories Inc, Prussia, PA, USA) and diluted with tap water. Sucrose (3\% w/v) solutions were 
prepared with D-sucrose (Thermo Fisher Scientific, Waltham, MA, USA) dissolved in tap water. CNO (supplied by the NIDA Drug Supply Program) was dissolved in DMSO (1\% v/v final concentration) and then diluted with $0.9 \%$ saline with a final concentration of $3 \mathrm{mM}$. Vehicle consisted of the same solution used to dissolve the $\mathrm{CNO}$ compound. Microinjections of $\mathrm{CNO}$ and vehicle were infused over the course of one minute $(0.3 \mu 1 / \mathrm{min})$ and injectors remained in the same place for at least one-minute post-infusion for diffusion of the drug and to help minimize back flow as the injectors are being removed. This injection volume and rate has been consistently used in site-directed injection methods within similar experiments (Rinker et al., 2017).

Surgical Procedures. Surgery methods were similar to those in our previous study with CRF+ neurons within the BNST (Rinker et al., 2017). Briefly, mice were anesthetized with a 1.5 $\mathrm{g} / \mathrm{kg}$ dose of ketamine/xylazine cocktail (100 and $10 \mathrm{mg} / \mathrm{kg}$, respectively) before beginning surgery procedures. Mice were then randomly assigned to receive bilateral $0.5 \mu 1 /$ side injections of either a Cre-dependent Gi/o-coupled DREADD (AAV8-hSyn-DIO-hM4d-mCherry) or a Credependent control virus (AAV8-hSyn-DIO-mCherry) (Addgene.org, Cambridge, MA, USA) in the dlBNST (with respect to bregma; AP: $+0.30 \mathrm{~mm}, \mathrm{ML}: \pm 1.10 \mathrm{~mm}$, DV: $-4.35 \mathrm{~mm}$ ) at a rate of $0.1 \mu \mathrm{l} / \mathrm{min}$ The injectors remained in place for at least 10-15 minutes following injections in an effort to minimize backflow of the virus through the syringe tract. Simultaneously, bilateral cannulae (Plastics One, Anaheim, CA, USA) were implanted into the VTA (with respect to bregma; AP: $-3.1 \mathrm{~mm}$, ML: $\pm 0.5 \mathrm{~mm}$, DV: $-4.5 \mathrm{~mm}$ ) of all animals with the assistance of Lecia Angle Two Stereotax (Lecia Biosystems, Buffalo Grove, IL, USA). Mice then remained in homecage for at least 5-6 weeks in order to let the virus incorporate into the GABAergic neurons and terminals before beginning the behavioral drinking portions of the experiment. 
Drinking-in-the-dark Procedures. A 4-day ON/3-day OFF DID protocol was used to model binge-like EtOH consumption in mice. Water bottles were removed and replaced with bottles containing 20\% EtOH (v/v) for 2-hours approximately 3-hours into the dark cycle. Day 4 was considered a test day where animals were microinjected with either $900 \mathrm{pmol} / \mathrm{side} \mathrm{CNO}$ or vehicle, using a Latin Square design, 30 minutes before a 2-hour EtOH drinking session where drinking was recorded every hour. Animals then went through a 3-day abstinence with only water ad lib. The full 7-days is considered a cycle of the DID exposure.

Animals went through two cycles of the DID procedure with EtOH and, after a week of additional abstinence, two additional cycles of DID were conducted with a 3\% sucrose (w/v) solution replacing the EtOH solution. Tail blood samples were taken at the end of the test day during both cycles of EtOH exposure in order to analyze the blood ethanol concentration (BEC) for each animal. BECs were used to determine whether or not the animal was consuming EtOH in a binge-like pattern (at least $80 \mathrm{mg} / \mathrm{dl}$ ) during the test day.

Tissue Preparation and IHC Procedures. Animals were sacrificed by a ketamine/xylazine cocktail, at a 10:1 ratio as before, before transcardial perfusion with PBS at a rate of $2.5 \mathrm{~mL} / \mathrm{min}$ for 4 minutes followed by a $4 \%$ paraformaldehyde solution at a rate of 2.5 $\mathrm{mL} / \mathrm{min}$ for 7 minutes; sufficient to flush and fix brain tissue. Brains were extracted and allowed to set in the $4 \%$ paraformaldehyde solution in order to further fix the tissue for 48 -hours before being transferred to PBS until tissue processing. Fixed brain tissue was cut into 40 micron slices using a Leica VT1000 S Vibratome (Lecia Biosystems, Buffalo Grove, IL, USA) and stored in cryopreserve solution. Using every fourth slice of tissue, IHC procedures were conducted in order to label CRF protein within the BNST.

Tissue slices were exposed to the primary Anti-CRF Rabbit antibody (Abcam Inc., Cambridge, MA, USA; ab8901) at a 1:250 concentration in the blocking solution for 48 to 72 
hours after an antigen retrieval at $65^{\circ} \mathrm{C}$ for 30 minutes in Antigen Retrieval Citra $1 \mathrm{X}$ Buffer to facilitate a more substantial binding potential (Garcia-Moreno et al., 2010; Cuevas Guaman et al., 2014). This concentration was determined by running a pilot concentration dilution experiment with tissue used in a previous study to determine proper primary concentration and antigen retrieval protocol. We then used a solution containing the secondary fluorescent antibody Donkey Anti-Rabbit Alexa Flour 488 (Abcam Inc., Cambridge, MA, USA) at a 1:1000 concentration in order to attach a green label to the primary antibody for visualization. These rinses made sure that all excess antibodies are rinsed away for maximum qualification of labeled neurons.

Tissue was then mounted onto glass slides and Vectashield Hard Set Medium containing DAPI (Vector Laboratories, Burlingame, CA, USA) was used to mount the coverslips over the tissue. This allowed for easy visualization of cell bodies that are co-expressing both the mCherry virus tag and the green-fluorescing CRF antibody tag. A Leica DM6000 FS was used to visualize DREADD virus and neuronal body fluorescence. A Zeiss LSM 800 confocal microscope (Zeiss, United States of America) was used to image tissue and allow 3D and z-stack imaging in order to fully determine whether co-localization is present within the BNST GABAergic projection neuron population as well as visualize terminals. Images were then analyzed in order to quantify the overall percentage of CRF+ cells overlapped with the overall DREADD+ neurons within the dIBNST. This was done using the cell counting function within ImageJ (Madison, WI, USA).

Statistical Analysis. Ethanol consumption and BECs achieved during DID procedures at the 2-hr time-point data were analyzed using a repeated-measures MANOVA with factors of treatment and treatment order to ensure there were no drug carryover or order effects. Separate hour time points used Bonferroni corrected paired and unpaired t-tests in order to uncover significant effects 


\section{Results}

\section{Anterograde Tracing of fibers Reveals VTA-projecting GABAergic Pathway Stemming}

from the dIBNST. Visualization of AAV8-hSyn-DIO-hM4d-mCherry terminals within the VTA

of VGat-ires-Cre mouse line confirmed an anterograde, GABAergic projection from the original

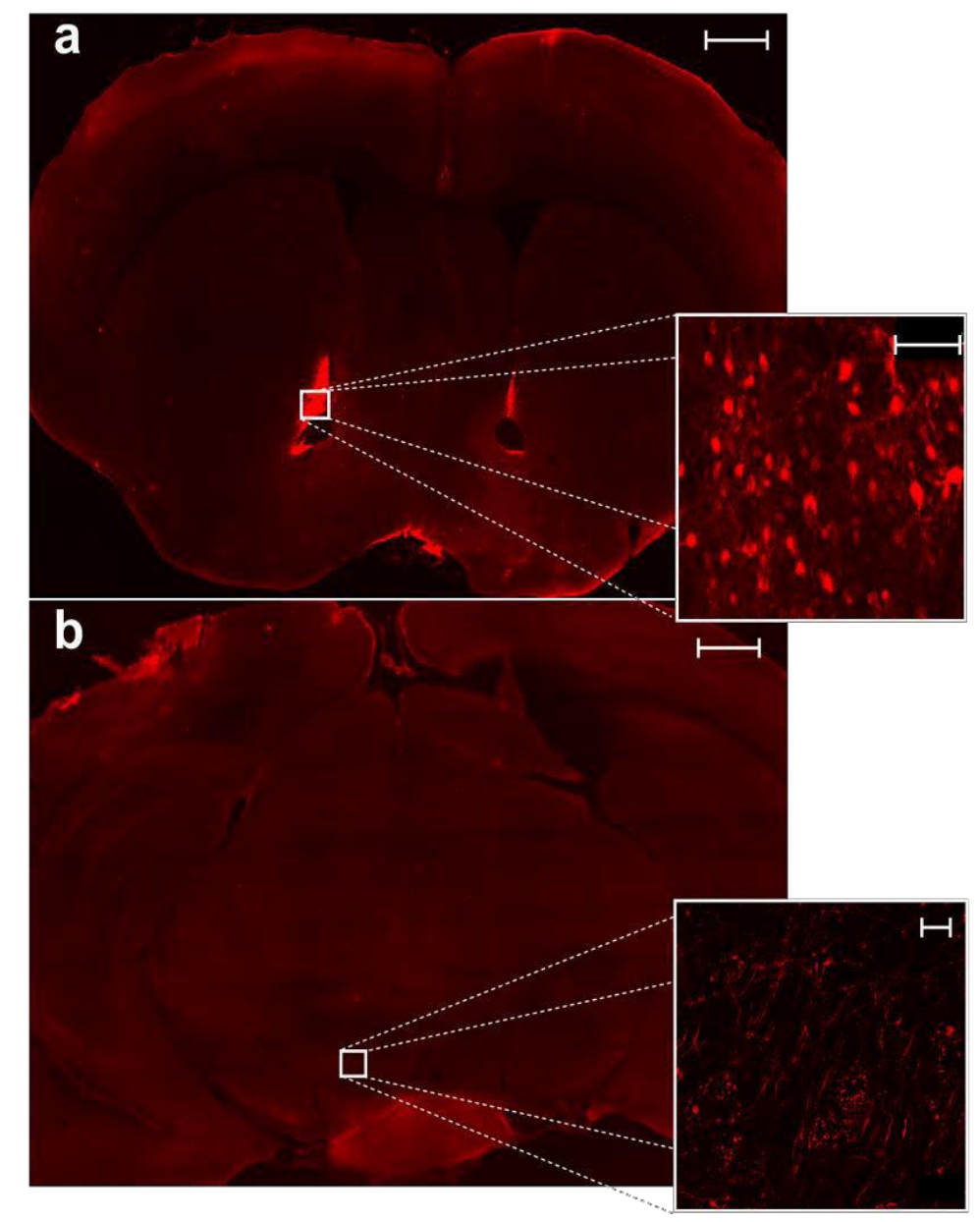

Figure 1: Tracing of GABAergic neuron terminals from the dIBNST to the VTA within the VGat-ires-Cre mouse line. A) Visualization of mCherry-tagged DREADD viral transfection within the dlBNST at $5 \mathrm{x}$ magnification $($ Scale bar $=500 \mu \mathrm{m})$ and visualization of DREADD-expressing cell bodies at 40x magnification (Scale bar $=75 \mu \mathrm{m}$ ). B) Innvervation of the VTA by dlBNST GABAergic fibers at $5 \mathrm{x}$ magnification $($ Scale bar $=500 \mu \mathrm{m})$ and visualization of terminal fiber at $64 \mathrm{x}$ magnification $($ Scale bar $=$ $10 \mu \mathrm{m})$

injection site of the dlBNST after 6 weeks of incubation (Fig. 1A, mCherry+ neurons in the dlBNST; Fig. 1B, mCherry+ terminals is the VTA). 


\section{Inhibition of GABAergic VTA-projecting dIBNST Neurons Reduces Binge-like Ethanol}

Consumption. Fig. 2 show data from the dlBNST $\rightarrow$ VTA silencing study during binge-like

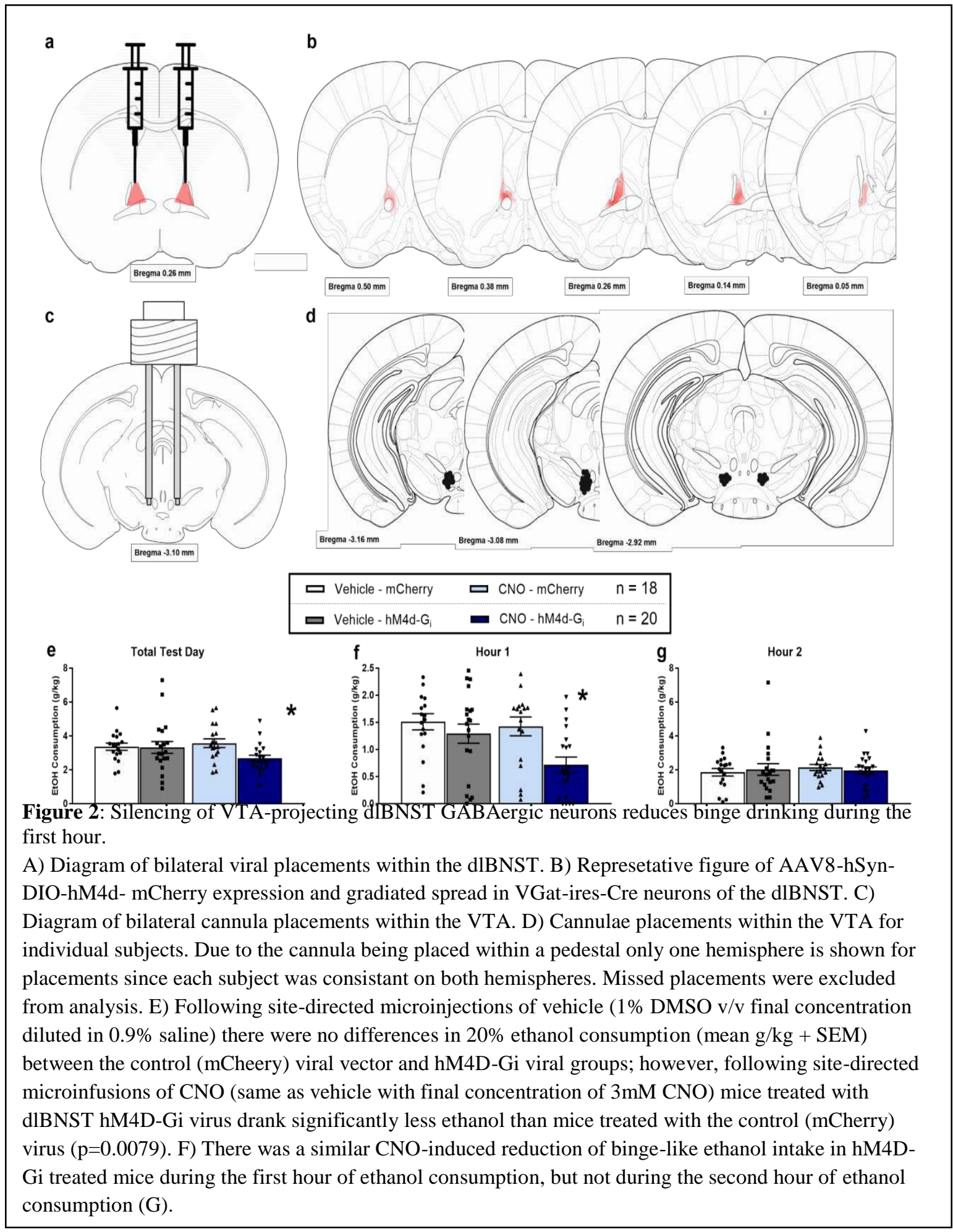

ethanol consumption. Fig. 2A shows a schematic representation of the viral-vector injection sites 
in the dlBNST, Fig. 2B depicts the average spread of the DREADD construct in the dlBNST in VGat-ires-Cre mice given dlBNST injection of Cre-dependent viral vector, verifying the expression of viral vector that was confined to the dlBNST. Critically, previous work has functionally validated the hM4D-Gi DREADD when traduced into the dlBNST by showing that CNO application significantly blunted neuronal activity in in vGat-ires-cre mice (Mazzone et al., 2018). Fig. 2C shows a schematic representation of the cannula placement in the VTA while Fig. 2D shows a schematic depicting the termination sites of cannulae implanted into the VTA of individual subjects. There were 6 animals removed from analysis due to incorrect placement of cannula. A repeated-measures (RM) MANOVA with within groups factors (hour and drug) and between groups factors (virus and sex) performed on total ethanol consumption indicated a main effect of hour $[F(1,34)=23.982, p<0.001]$ and interaction of drug $x$ virus $[F(1,34)=5.883$, $\mathrm{p}=0.021]$ and drug $x$ sex $[\mathrm{F}(1,34)=13.866, \mathrm{p}=0.001 ;$ male $\mathrm{CNO}=1.72+0.10$, male vehicle $=$ $1.53+0.13$, female $\mathrm{CNO}=1.36+0.11$, female vehicle $=1.86+0.15]$. The drug $\mathrm{x}$ sex interaction reflected the tendency for male mice to drink more ethanol when treated with CNO, while the female mice tended to drink more ethanol when treated with vehicle. A further analysis drug $\mathrm{x}$ virus interaction using the planned-comparison Bonferroni's correction t-test (with significance set at $\mathrm{p}<0.008)$ revealed a significant difference between the AAV8-hSyn-DIOhM4d-mCherry virus and the AAV8-hSyn-DIO-mCherry virus groups that were treated with CNO $[\mathrm{p}=0.0079]$. There were no differences between virus conditions in mice treated with vehicle (Fig. 2E). Similarly, a significant difference emerged between the AAV8-hSyn-DIOhM4d-mCherry virus and the AAV8-hSyn-DIO-mCherry virus groups that were treated with CNO at the 1-hoour consumption measure [ $\mathrm{p}=0.003$, with no differences between virus conditions in mice treated with vehicle (Fig. 2F). There were no group differences when the 2 nd hour of consumption were analyzed (Fig. 2G). Finally, analysis of BEC data failed toreveal 
significant effects (control virus-vehicle, $110 \pm 13.84 \mathrm{mg} / \mathrm{dl}$; control virus-CNO, $131.5 \pm 13.66 \mathrm{dl}$;

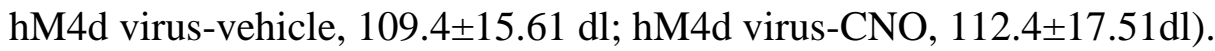

\section{Inhibition of GABAergic VTA-projecting dIBNST Neurons does not Influence Sucrose}

Consumption. Fig. 3 show data from the dlBNST $\rightarrow$ VTA silencing study during binge-like sucrose consumption. A repeated-measures (RM) MANOVA with within groups factors(hour
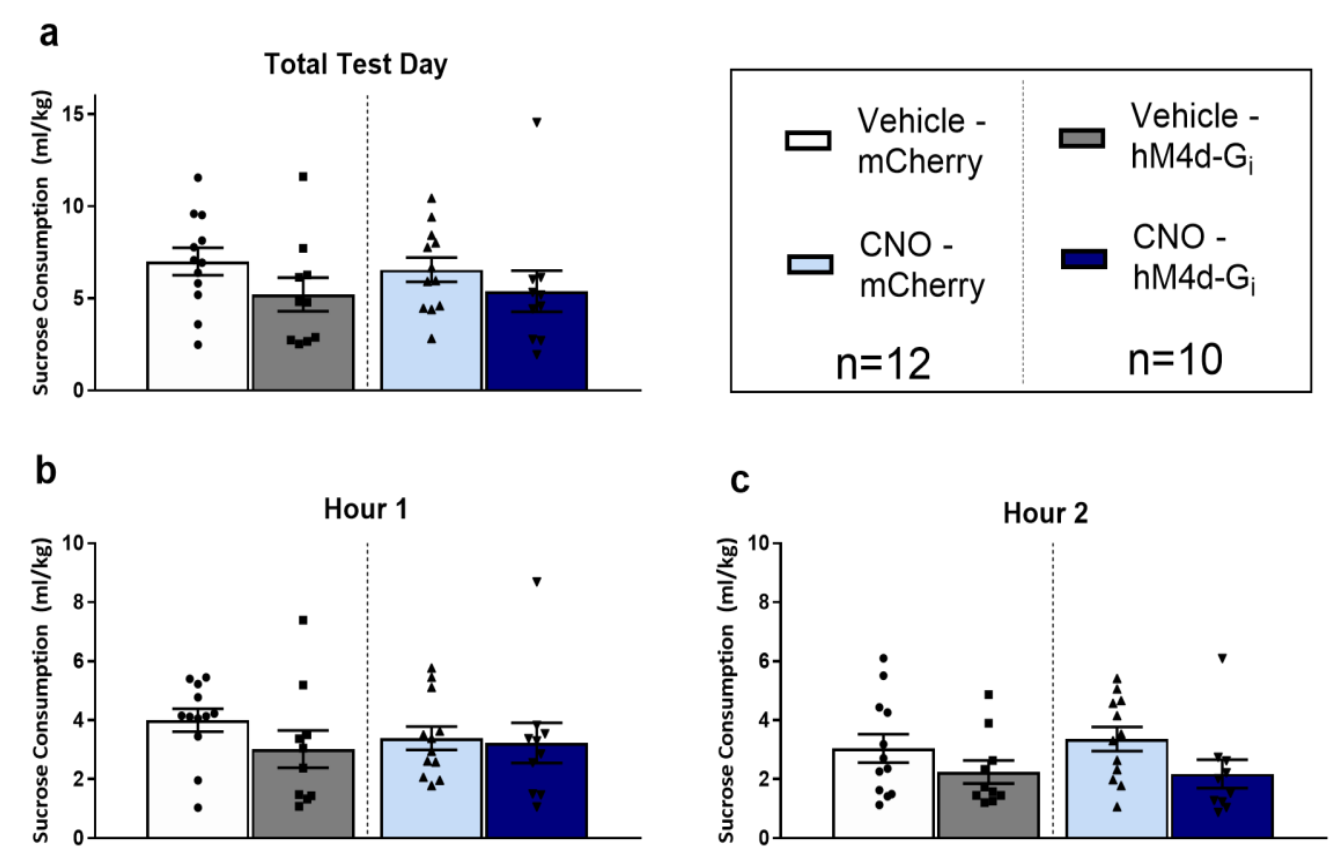

Figure 3: Silencing of VTA-projecting dlBNST GABAergic neurons had no significant effect on binge drinking of $3 \%$ sucrose. A) There was no significant reduction of $3 \%$ sucrose drinking within any of the 4 groups during the total 2-hour binge test. B) There was no significant effect of virus or drug treatment on sucrose drinking during the first hour of sucrose drinking. C) There was also no reduction of sucrose drinking during the second hour of sucrose consumption.

and drug) and between groups factors (virus and sex) performed on 2-hour sucroseconsumption data failed to produce any main effects or interaction effects (Fig. 3A). Further, there were no significant differences during the 1st (Fig. 3B) or 2nd (Fig. 3C) hours of sucroseconsumption. 


\section{Co-localization of CRF immunoreactivity with Cre-dependent DREADD in in the dIBNST}

of VGat-ires-Cre mice. Fig. 4 shows confocal imaging of AAV8-hSyn-DIO-hM4d-mCherry

(red) with anti-CRF in Alexaflour-488 (green) and cell nuclei DAPI staining (blue) at 20x (Fig.

4A) and 63x (Fig. 4B) magnification. A qualitative assessment of the co-expression suggests that most, if not all, of the AAV8-hSyn-DIO-hM4d-mCherry+ neurons also expressed CRF immunoreactivity, consistent with the observation that $98 \%$ of GABAergic neurons in the dlBNST entail a CRF phenotype (Nguyen et al., 2016).

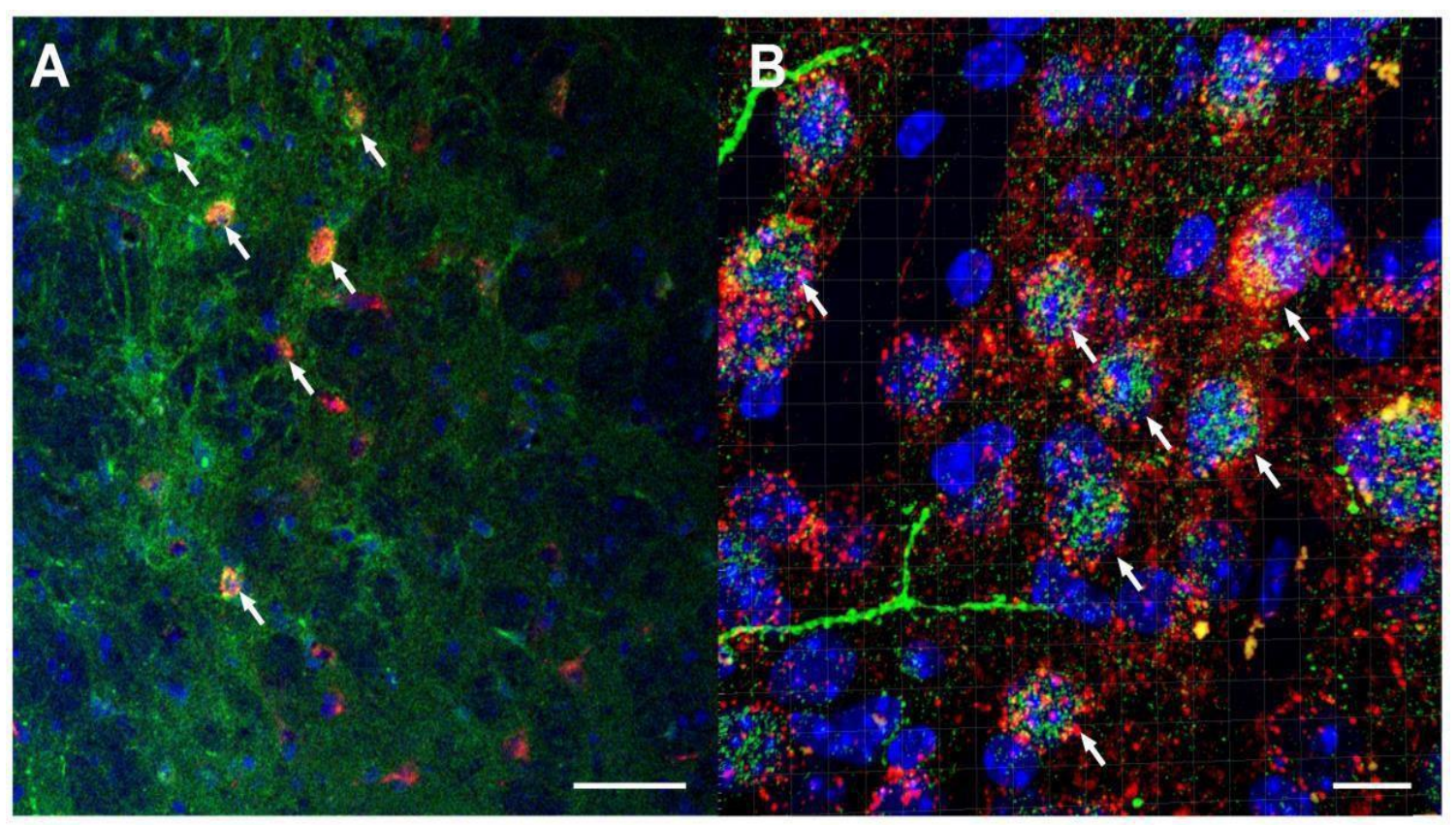

Figure 4: CRF IHC co-localization with Cre-dependent AAV8-hSyn-DIO-hM4d-mCherry in VGat-ires-Cre mice.

A) Confocal imaging of AAV8-hSyn-DIO-hM4d-mCherry viral vector (red) with anti-CRF in Alexaflour488 (green) and cell nuclei DAPI staining (blue) at 20x magnification. White arrows emphasize colocalized neurons (scale bar $=50 \mu \mathrm{m}$ ). B) Confocal, $\mathrm{z}$-stacked 3D reconstructed of co-localization of AAV8-hSyn-DIO-hM4d- mCherry virus (red) with CRF (green) and DAPI (blue) at 63x magnification. White arrows emphasize co-localized neurons (scale bar $=10 \mu \mathrm{m})$.

\section{Discussion}

Here we show that silencing VTA-projecting GABAergic dIBNST neurons significantly reduced binge-like EtOH intake, particularly during the first hour of the 2-hour EtOH consumption test. Importantly, $\mathrm{CNO}$ did not alter binge-like ethanol intake relative to vehicle treatment in mice expressing the control viral vector, ruling out potential off-target effects of $\mathrm{CNO}$ with respect to 
ethanol intake. There was also no effect of virus or drug on sucrose drinking using the DID paradigm, suggesting that the effect of silencing VTA-projecting GABAergic dIBNST neurons was specific to EtOH consumption and did not influence the consumption of another salient, natural reinforcer. The IHC and confocal microscopy revealed GABAergic neurons that also colocalized with the CRF peptide, reaffirming our previous findings showing blunted binge-like ethanol intake with silencing of VTA-projection dlBNST neurons expressing CRF (Rinker et al., 2017). When taken together, our results demonstrate that dlBNST GABAergic/CRF+ circuitry projecting to the VTA may be the population that is causing this in the modulation of binge-like EtOH drinking.

We have previously shown that silencing VTA-projecting CRF+ neurons within the dIBNST significantly blunted binge-like EtOH intake (Rinker et al., 2017). A recent study found that while CRF+ neurons in the BNST co-express both glutamate and GABA, within the dlBNST, about 98\% of CRF+ neurons are GABAergic (Nguyen et al., 2016). Together, these findings suggest that our previous study using CRF-ires-Cre mice may have stemmed from the silencing of a GABAergic population of neurons within the dlBNST. The observation by Nguyen et al. (2016) that a population of BNST glutamatergic neurons also co-express a CRF+ phenotype, and research showing that a population of BNST glutamatergic neurons innervates the VTA and have effects on consummatory behavior (Jennings et al., 2013) together highlight the importance of future research to investigate the role of VTA-projecting glutamatergic BNST neurons in the modulating of binge-like ethanol intake. However, given that $98 \%$ of $\mathrm{CRF}+$ neurons in the dIBNST are co-expressed with a GABAergic phenotype, the contributions of a VTA-projecting glutamatergic dlBNST circuit in modulating binge-like ethanol intake seems minimal. Recent evidence has suggested that $\mathrm{CNO}$ may back-metabolize to clozapine, which is a psychoactive drug that may act as a potential confound to chemogenetic studies (MacLaren et al., 
2016). However, there are two observations within our study that placate this concern: First, CNO had no effect, relative to vehicle treatment, in mice that expressed the control viral vector. Second, there was no effect of CNO on sucrose consumption in either the AAV8-hSyn-DIOhM4d-mCherry or control virus groups. Together, these observations rule out the likely possibility that off-target effects from the use of $\mathrm{CNO}$ influenced binge-like ethanol intake. There is also the concern that CNO may be flowing outside of the VTA region and possibly effecting other possible projections from the BNST where the viral vector was injected. While the only way to be sure that this could not be happening would be to use a tagged $\mathrm{CNO}$ in order to visualize the area of effect, previous studies using this exact injection volume and rate have had a fairly accurate diffusion of drug.

Interestingly, Rinker et al. (2017) found that site-directed infusion of a CRF-1 receptor (CRF1R) antagonist into the VTA blunted binge-like ethanol consumption, but that while using simultaneous infusion of CRF1R and CRF2R antagonists the paradigm failed to alter intake. Thus, intact CRF2R signaling in the VTA is necessary for CRF1R antagonism to blunt bingelike ethanol intake. Rinker et al. presented a theoretical model whereby GABAergic transmission from VTA-projecting dIBNST neurons promotes binge-like ethanol intake, and that GABAergic signaling from these neurons is facilitated or inhibited by CRF1R or CRF2R, respectively. Further, they postulate that blockade of CRF1R "unmasks" the ability of CRF2R to blunt GABAergic signaling, resulting in reduced binge intake. Blockade of both receptors simultaneous negates this effect, leaving GABAergic signaling unregulated which is consistent with the observed unaltered binge-like drinking when both CRF receptor antagonist are infused into the VTA. The present results provide additional support for this theoretical model by directly demonstrating the central role of GABAergic signaling in this circuit in the modulating of binge-like ethanol intake. This pharmacology along with pharmacological studies showing 
GABA-A receptor inhibition in the posterior VTA reduced binge-like EtOH consumption in C57BL/6J mice (Melón \& Boehm, 2011) implies and importance in focusing on this co-release of CRF and GABA in order to fully understand their interaction within the VTA, and particularly this dIBNST innervation within this region. This is an significant future direction for our work with this circuit.

In summary, we show that inhibition of a VTA-projecting GABAergic circuit stemming fromthe dlBNST reduces binge-like ethanol drinking, suggesting that this circuit plays a critical role in modulating binge-like EtOH consumption. This effect was specific to EtOH consumption, as silencing this circuit had no impact on the consumption of the natural reinforcer, sucrose. We also show that the GABAergic population under investigation within the dIBNST co-expresses CRF. Overall, this study and Rinker et al. (2017) emphasize the importance of VTA-projecting GABAergic/CRF+ neurons from the dlBNST in the early stages of binge-like EtOH intake. Neuroadaptations in this circuit may be an important component of increased voluntary consumption of ethanol stemming from repeated bouts of binge drinking (Cox et al., 2013), thus further characterization of this neurocircuitry may provide valuable insight into the mechanisms underlying the development of alcohol use disorders.

\section{Acknowledgments}

We gratefully acknowledge support from the National Institute on Alcoholism and Alcohol Abuse (NIAAA; R01 AA022048, R01 AA013573, \& R01 AA015148) and the National Institute on Drug Abuse (NIDA; T32 DA00724426). CNO was supplied by the NIDA Drug Supply Program. 


\section{REFERENCES}

Cox, B.R., Olney, J.J., Lowery-Gionta, E.G., Sprow, G.M., Rinker, J.A., Navarro, M., Kash, T.L. \& Thiele, T.E. (2013) Repeated Cycles of Binge-Like Ethanol (EtOH)- Drinking in Male C57BL/6J Mice Augments Subsequent Voluntary EtOH Intake But Not Other Dependence-Like Phenotypes. Alcoholism: Clinical and Experimental Research, 37, 1688-1695.

Crabbe, J.C., Harris, R.A. \& Koob, G.F. (2011) Preclinical studies of alcohol binge drinking. Annals of the New York Academy of Sciences, 1216, 24-40.

Cuevas Guaman, M., Sbrana, E., Shope, C., Showalter, L., Hu, M., Meloche, S. \& Aagaard, K. (2014) Administration of antenatal glucocorticoids and postnatal surfactant ameliorates respiratory distress syndrome-associated neonatal lethality in Erk3(-/-) mouse pups. Pediatric research, 76, 24-32.

Dabrowska, J., Hazra, R., Guo, J., DeWitt, S. \& Rainnie, D. (2013) Central CRF neurons are not created equal: phenotypic differences in CRF-containing neurons of the rat paraventricular hypothalamus and the bed nucleus of the stria terminalis. Frontiers in Neuroscience, 7.

Garcia-Moreno, F., Pedraza, M., Di Giovannantonio, L.G., Di Salvio, M., Lopez-Mascaraque, L., Simeone, A. \& De Carlos, J.A. (2010) A neuronal migratory pathway crossing from diencephalon to telencephalon populates amygdala nuclei. Nature neuroscience, 13, 680-689.

Jennings, J.H., Sparta, D.R., Stamatakis, A.M., Ung, R.L., Pleil, K.E., Kash, T.L. \& Stuber, G.D. (2013) Distinct extended amygdala circuits for divergent motivational states. Nature, 496, 224228.

Kudo, T., Uchigashima, M., Miyazaki, T., Konno, K., Yamasaki, M., Yanagawa, Y., Minami, M. $\&$ Watanabe, M. (2012) Three types of neurochemical projection from the bed nucleus of the stria terminalis to the ventral tegmental area in adult mice. The Journal of neuroscience, 32, 18035-18046.

Li, T.K., Hewitt, B.G. \& Grant, B.F. (2007) The Alcohol Dependence Syndrome, 30 years later: a commentary. the 2006 H. David Archibald lecture. Addiction (Abingdon, England), 102, 152215 
Lowery-Gionta, E.G., Navarro, M., Li, C., Pleil, K.E., Rinker, J.A., Cox, B.R., Sprow, G.M., Kash, T.L. \& Thiele, T.E. (2012) Corticotropin Releasing Factor Signaling in the Central Amygdala is Recruited during Binge-Like Ethanol Consumption in C57BL/6J Mice. The Journal of Neuroscience, 32, 3405-3413.

Lowery, E.G. \& Thiele, T.E. (2010) Pre-clinical evidence that corticotropin-releasing factor (CRF) receptor antagonists are promising targets for pharmacological treatment of alcoholism. CNS \& neurological disorders drug targets, 9, 77-86.

MacLaren, D.A.A., Browne, R.W., Shaw, J.K., Krishnan Radhakrishnan, S., Khare, P., España, R.A. \& Clark, S.D. (2016) Clozapine N-Oxide Administration Produces Behavioral Effects in Long-Evans Rats: Implications for Designing DREADD Experiments. eneuro, 3.

Marshall, S.A., Rinker, J.A., Harrison, L.K., Fletcher, C.A., Herfel, T.M. \& Thiele, T.E. (2015) Assessment of the Effects of 6 Standard Rodent Diets on Binge-Like and Voluntary Ethanol Consumption in Male C57BL/6J Mice. Alcoholism: Clinical and Experimental Research, 39, 1406-1416.

Mazzone, C.M., Pati, D., Michaelides, M., DiBerto, J., Fox, J.H., Tipton, G., Anderson, C., Duffy, K., McKlveen, J.M., Hardaway, J.A., Magness, S.T., Falls, W.A., Hammack, S.E., McElligott, Z.A., Hurd, Y.L. \& Kash, T.L. (2018) Metabolic mapping of downstream network activity following CNO-induced activation of hM3Dq in BNST VGAT neurons. Molecular psychiatry, $23,1$.

Melón, L.C. \& Boehm, S.L. (2011) GABAA receptors in the posterior, but not anterior, ventral tegmental area mediate Ro15-4513-induced attenuation of binge-like ethanol consumption in C57BL/6J female mice. Behavioural Brain Research, 220, 230-237.

Nguyen, A.Q., Dela Cruz, J.A., Sun, Y., Holmes, T.C. \& Xu, X. (2016) Genetic cell targeting uncovers specific neuronal types and distinct subregions in the bed nucleus of the stria terminalis. The Journal of comparative neurology, 524, 2379-239 
Rhodes, J.S., Best, K., Belknap, J.K., Finn, D.A. \& Crabbe, J.C. (2005) Evaluation of a simple model of ethanol drinking to intoxication in C57BL/6J mice. Physiology \& behavior, 84, 53-63.

Rinker, J.A., Marshall, S.A., Mazzone, C.M., Lowery-Gionta, E.G., Gulati, V., Pleil, K.E., Kash, T.L., Navarro, M. \& Thiele, T.E. (2017) Extended Amygdala to Ventral Tegmental Area Corticotropin-Releasing Factor Circuit Controls Binge Ethanol Intake. Biological Psychiatry, 81, 930-940.

Roberto, M., Cruz, M.T., Gilpin, N.W., Sabino, V., Schweitzer, P., Bajo, M., Cottone, P., Madamba, S.G., Stouffer, D.G., Zorrilla, E.P., Koob, G.F., Siggins, G.R. \& Parsons, L.H. (2010) Corticotropin releasing factor-induced amygdala gamma-aminobutyric Acid release plays a key role in alcohol dependence. Biol Psychiatry, 67, 831-839.

Rogan, S.C. \& Roth, B.L. (2011) Remote control of neuronal signaling. Pharmacological reviews, 63, 291-315.

Roth, B.L. (2016) DREADDs for Neuroscientists. Neuron, 89, 683-694.

Stamatakis, A.M., Sparta, D.R., Jennings, J.H., McElligott, Z.A., Decot, H. \& Stuber, G.D. (2014) Amygdala and Bed Nucleus of the Stria Terminalis Circuitry: Implications for addictionrelated behaviors. Neuropharmacology, 76, 10.1016/j.neuropharm.2013.1005.1046.

Thiele, T.E., Crabbe, J.C. \& Boehm, S.L., 2nd (2014) "Drinking in the Dark" (DID): a simple mouse model of binge-like alcohol intake. Curr Protoc Neurosci, 68, 949 41-12.

Thiele, T.E. \& Navarro, M. (2014) "Drinking in the dark" (DID) procedures: a model of bingelike ethanol drinking in non-dependent mice. Alcohol, 48, 235-241.

Vong, L., Ye, C., Yang, Z., Choi, B., Chua, S. \& Lowell, B.B. (2011) Leptin Action on GABAergic Neurons Prevents Obesity and Reduces Inhibitory Tone to POMC Neurons. Neuron, $71,142-154$. 